\title{
Migración: retos y oportunidades desde la perspectiva de los Objetivos de Desarrollo Sostenible (ODS)
}

\section{Migration: challenges and opportunities from the perspective of Sustainable Development Goals (SDGs)}

\author{
Dra. Agrivalca Ramsenia Canelón Silva es profesora e investigadora de la Universidad de la Sabana (Colombia) \\ (https://orcid.org/0000-0002-6368-5268) (agrivalca.canelon@unisabana.edu.co)
}

Dra. Ana Almansa Martínez es profesora e investigadora de la Universidad de Málaga (España) (https://orcid.org/0000-0003-0256-6369) (anaalmansa@uma.es)

\begin{abstract}
Resumen
Al tenor del creciente consenso en torno a que una agenda para el desarrollo después de 2015 demanda el abordaje de la migración internacional contemporánea con el ánimo de reducir la pobreza y la desigualdad, a la par que combatir la discriminación, la exclusión y la marginalización, se describe, de modo sucinto, cómo los Objetivos de Desarrollo Sostenible (ODS), enmarcados en la Agenda 2030, visibilizan e incluyen a las migraciones y el refugio como temas de discusión. Para ello, se parte de una revisión documental en torno a las bases normativas y conceptuales que sustentan la relación entre la migración y el desarrollo, brindando luego un breve estatus de la situación migratoria en Iberoamérica con foco en América Latina y el Caribe -particularmente el caso de Venezuela y el éxodo por el que atraviesa en la actualidad como producto de la contracción económica, la crisis humanitaria y la conflictividad político-social-, para finalizar con una reflexión en torno a los actores convocados a hacer parte de este esfuerzo y las acciones que pueden desplegar de cara a aminorar la «migración involuntaria a gran escala» provocada por «conflictos, desastres o por motivos ambientales o económicos», la cual figura dentro de los 10 principales riesgos mundiales contemplados de manera reiterada en los informes publicados por el Foro Económico Mundial entre 2016 y 2018.
\end{abstract}

\begin{abstract}
In line with the growing consensus that a post in the 2015 development agenda demands the approach of contemporary international migration with the aim of reducing poverty and inequality, while fighting against discrimination, exclusion and marginalization, this article describes how the Sustainable Development Goals (SDGs), framed in the 2030 Agenda, make visible and include migrations and refugees as topics for discussion. To do so, the article begins with a literature review regarding the normative and conceptual bases of the relationship between migration and development, providing a brief status of the migration behavior flows in Ibero-America with a focus on Latin American and the Caribbean-particularly the case of Venezuela and the exodus it is currently experiencing as a result of economic contraction, humanitarian crisis and political and social conflict-, to end with a reflection on the actors invited to participate in this effort and the actions that can be deployed in order to reduce the "involuntary large-scale migration" caused by "conflicts, disasters or environmental or economic reasons", which is within the top 10 global risks repeatedly contemplated in the reports published by the World Economic Forum between 2016 and 2018. The article hopes to contribute to the debate in favor of migratory and integration policies with better planning and management, and real impact on the lives of migrants.
\end{abstract}

\section{Palabras clave I keywords}

Migración, refugiados, desplazados, objetivos de desarrollo sostenible, diásporas, derechos humanos. Migration, refugees, displaced persons, sustainable development goals, diasporas, human rights.

Cómo citar: Canelón Silva, A.R. y Almansa Martínez, A. (2018). Migración: retos y oportunidades desde la perspectiva de los Objetivos de Desarrollo Sostenible (ODS). Retos Revista de Ciencias de la Administración y Economía, 8(16), 109-120. https://doi.org/10.17163/ret.n16.2018.08 


\section{Introducción}

Las migraciones no constituyen un fenómeno nuevo. Casi siempre abandonar el lugar de origen o trasladarse a otro lugar de residencia, ya sea al interior de un país o a través de las fronteras internacionales, sobre todo tratándose de los habitantes de los países en desarrollo, puede ser la mejor, cuando no la única salida para aumentar las oportunidades de ingreso, o tener acceso a más servicios de educación y salud. En la toma de esta decisión, para nada exenta de incertidumbre, subyace la esperanza de combinar los talentos propios con los recursos del país de destino, para el beneficio personal, el de los familiares cercanos -quienes a menudo acompañan al migrante o, en su defecto, lo siguen después-, el de quienes se quedan atrás y el de la sociedad donde se establece el nuevo hogar (Castillo, 2015; PNUD, 2009).

No obstante, no pocas veces los migrantes suelen sufrir el rechazo de quienes temen a los «recién llegados», lo que evidencia el impacto de su presencia sobre las dimensiones culturales y las actitudes de las personas en las sociedades de recepción, dado el crecimiento observado en el volumen de individuos desplazados y refugiados durante los años recientes en razón de la inestabilidad política, la violencia delictiva, las persecuciones, los desastres naturales, la degradación ambiental vinculada al cambio climático, la pobreza y la desigualdad (El Vaticano, 2017). He aquí la irrupción de riesgos y vulnerabilidades a los que están expuestos buena parte de los migrantes; por ejemplo, los refugiados, quienes permanecen cerca del país del que huyen, viviendo habitualmente en campamentos hasta que la situación en casa permite retornar; o las víctimas del tráfico humano (acompañado de violencia y abuso sexual), especialmente mujeres jóvenes que son engañadas con promesas de mejores horizontes de vida (Foro Económico Mundial, 2018).

De acuerdo con el Global Forum on Migration and Development GFMD Turkey 2014-2015, hasta 2014 la cifra de migrantes internacionales había aumentado en aproximadamente un $32 \%$, alcanzando los 232 millones de personas, al cabo que las remesas enviadas por éstos a los países en desarrollo experimentaron un incremento de más de cinco veces, registrando una suma estimada en 436 mil millones de dólares estadounidenses. Entretanto, la cifra de desplazados forzosos a raíz de situaciones de crisis (refugiados y solicitantes de asilo) arribó a los 59,5 millones de personas.

Al día de hoy, el fenómeno migratorio se muestra todavía más complejo y permeable a lecturas negativas en virtud de la tensión natural planteada por la globalización a los Estados-nación westfalianos con soberanía plena para gestionar y controlar sus fronteras (Pinazo, 2017). De resultas, quienes desean o se ven obligados a migrar se topan, cada vez más, con obstáculos erigidos por los gobiernos para restringir, si acaso no cerrar la escala de entrada y desplazamiento, incluso en medio de la paradoja que supone la disminución de las barreras para el intercambio de bienes (PNUD, 2009).

Dentro de este panorama, por lo regular, las políticas en los países de destino suelen favorecer a las personas con más educación, estilándose la invitación a profesionales para instalarse con sus familias, o permitiendo a los estudiantes quedarse tan pronto consiguen titularse. Por el contrario, los trabajadores con baja calificación académica o pocos conocimientos especializados enfrentan más impedimentos, siendo relegados a la demanda de mano de obra en sectores como la agricultura, la construc- 
ción, la industria, la manufactura y los servicios, lo que genera gran controversia ante la opinión de que llenan «puestos vacantes», pero también desbancan a los trabajadores locales y hacen disminuir los salarios.

Con todo, los migrantes, independientemente de las razones que esgriman para irse del sitio del que son oriundos, merecen ser respetados en su dignidad, derechos y libertades, desprendiéndose la necesidad de ofrecer una respuesta conjunta a través de la cooperación internacional y la responsabilidad compartida para promover y adoptar medidas eficaces que protejan y contribuyan a la mejora de su situación junto a la de los refugiados, los solicitantes de asilo y los desplazados internos, evitando se conviertan en víctimas de redes criminales y tráfico de seres humanos, explotación y abuso (El Vaticano, 2017).

Es así como, al tenor del creciente consenso en torno a que una agenda para el desarrollo después de 2015 demanda el abordaje de la migración internacional contemporánea con el ánimo de reducir la pobreza y la desigualdad, a la par que combatir la discriminación, la exclusión y la marginalización, el presente artículo describe, de modo sucinto, cómo los Objetivos de Desarrollo Sostenible (ODS), enmarcados en la Agenda 2030, visibilizan e incluyen a las migraciones y el refugio como temas de discusión. Para ello, se parte de una revisión documental en torno a las bases normativas y conceptuales que sustentan la relación entre la migración y el desarrollo, brindando luego un breve estatus de la situación migratoria en Iberoamérica con foco en América Latina y el Caribe, para finalizar con una reflexión en torno a los actores convocados a hacer parte de este esfuerzo y las acciones que pueden desplegar.

\section{Migración, desarrollo y Agenda 2030}

Con el advenimiento del siglo XXI, la migración pasó a ocupar un sitial relevante en el marco de las políticas de la mayoría de los Estados miembros de la Organización de Naciones Unidas (ONU), constituyendo un hito en esta línea el «Diálogo de Alto Nivel sobre la Migración Internacional y el Desarrollo», cuya primera edición se celebró en septiembre de 2006. Este espacio brindó a los países participantes la posibilidad de abordar la migración desde aristas multidimensionales, con el fin de determinar los mecanismos idóneos para maximizar sus beneficios de cara al desarrollo y minimizar sus repercusiones negativas. De allí la creación del Foro Mundial sobre Migración y Desarrollo en tanto plataforma de carácter voluntario y no vinculante, destinada fundamentalmente a la cooperación, junto al Grupo Mundial sobre Migración, abocado a funciones de seguimiento en cuanto a la aplicación de instrumentos y normas internacionales y regionales, además del impulso de enfoques interinstitucionales coherentes, integrales y coordinados.

Esta iniciativa sería refrendada por el II Diálogo de Alto Nivel sobre la Migración Internacional y el Desarrollo: Conseguir que la migración funcione, llevado a cabo el 3 y 4 de octubre de 2013 al amparo del $68^{\circ}$ período de sesiones anual de la Asamblea General de la Organización de Naciones Unidas (ONU). La declaración derivada de este encuentro puso de relieve el carácter de la movilidad humana como impulsor crucial para el desarrollo sostenible, subrayando su contribución al logro de los Objetivos de Desarrollo del Milenio (ODM), de donde se sigue el exhorto explícito a los Estados para que defiendan 
los Derechos Humanos de los migrantes, los refugiados y los desplazados internos, y garanticen a éstos el goce de las libertades fundamentales.

De esta manera, la Declaración define el contexto y el tono de la Agenda post 2015, erigiéndose sin duda en una aportación decisiva para la Agenda 2030 sobre la base de un plan de acción que abarca 17 objetivos y 169 metas encaminados a la atención de los desafíos que comportan las tres dimensiones del desarrollo sostenible -económica, social y ambiental- a la luz de una visión global, integral y equilibrada en procura de erradicar la pobreza, reducir la desigualdad, proteger el planeta y asegurar la prosperidad para todos. Asimismo, incluye el compromiso de cooperar a nivel internacional para conseguir que la migración sea ordenada, segura y regular, reafirmando la promesa de «no dejar a nadie atrás».

Con arreglo a esta tónica, la Agenda 2030 para el Desarrollo Sostenible fue aprobada por Jefes de Estado y altos representantes de Gobierno durante la reunión de la Asamblea General de la Organización de Naciones Unidas (ONU) realizada en septiembre de 2015, entrando en vigor el $1^{\circ}$ de enero de 2016. A este primer paso, le siguió el informe del Secretario General del organismo, titulado En condiciones de seguridad y dignidad: respuesta a los grandes desplazamientos de refugiados y migrantes, publicado en abril de 2016, el cual allanó la ruta de una Cumbre Extraordinaria en septiembre de ese mismo año, traducida a la postre en la Declaración de Nueva York, en cuyo nombre los Estados miembros de la Organización de Naciones Unidas (ONU) acordaron compartir la responsabilidad de acoger y prestar ayuda a los migrantes, con el ánimo de concretar, en 2018, la aprobación de un «Pacto Mundial sobre Migración» en aras de configurar un sistema de gobernanza mundial para acometer la movilidad humana (OIM, 2017: I).

A la sazón, el documento llama a una lectura multifactorial de la migración desde una perspectiva compleja que, articulada con la dinámica de los Objetivos de Desarrollo Sostenible (ODS), reconoce las necesidades de los migrantes en calidad de grupo vulnerable que precisa empoderamiento. Así, aunque la movilidad humana no aparece establecida como un objetivo específico o una meta concreta, transversalmente puede advertirse como un campo explícito alrededor del que se aglutinan temas en los que se inscribe la actuación política, a saber:

a) La protección de los Derechos Humanos por encima de otros factores culturales o de nacionalidad.

b) La lucha contra las redes organizadas de tráfico y trata de personas, dado el alto componente de feminización de los flujos migratorios.

c) La implementación de medidas urgentes por lo que atañe a la escolarización inmediata de los infantes como primera medida de inserción social (programas de acceso a la educación primaria y secundaria).

d) El fomento de una cultura de acogida en contraposición a las expresiones de xenofobia y racismo.

e) El apoyo a los principales países receptores de los migrantes y refugiados.

f) El despliegue de políticas de vivienda.

g) El carácter excepcional y temporal de los campos de refugiados. 
h) El compromiso de ampliar los cauces legales para el reasentamiento.

i) El análisis científico de datos que permitan delimitar las coordenadas de las migraciones y la comprensión de sus causas (Fernández et. al, 2017).

A partir de estas consideraciones, y yendo al detalle, en el desglose de los objetivos y las metas de la Agenda 2030 para el Desarrollo Sostenible, la migración encuentra cabida y referencia tal como se plasma a continuación:

- $\quad$ Objetivo 1: Fin de la pobreza.

Meta 1.3. Poner en práctica sistemas de protección social, incluidos niveles mínimos, y lograr una cobertura sostenible de los pobres y vulnerables.

Meta 1.5. Fortalecer la resiliencia de los pobres y de las personas más expuestas a crisis y desastres económicos, sociales y ambientales.

- Objetivo 3. Salud y bienestar.

Meta 3.8. Lograr la cobertura sanitaria universal.

Meta 3.c. Aumentar la financiación de la salud y formar personal sanitario que dé abasto en los países en desarrollo.

Meta 3.d. Reforzar la capacidad de los países en materia de alerta temprana, reducción de riesgos y gestión de los riesgos para la salud nacional y mundial.

- Objetivo 4. Educación de calidad.

Meta 4.b. Promoción de becas para oportunidades de educación internacional (movilidad estudiantil).

- Objetivo 5. Igualdad de Género.

Meta 5.2. Eliminar todas las formas de violencia contra las mujeres y las niñas.

Meta 5.6. Garantizar el acceso universal a la salud sexual y reproductiva y los derechos reproductivos.

- Objetivo 8. Trabajo decente y crecimiento económico.

Meta 8.7. Erradicar el trabajo forzoso, poner fin a las formas modernas de esclavitud y la trata de personas; eliminar el trabajo infantil.

Meta 8.8. Proteger los derechos laborales y promover un entorno de trabajo seguro y sin riesgos para todos los trabajadores, incluidos los trabajadores migrantes.

- Objetivo 10. Reducción de las desigualdades.

Meta 10.7. Facilitar la migración ordenada y segura mediante políticas migratorias bien gestionadas.

Meta 10.c. Reducción de los costos de las remesas.

- Objetivo 11. Ciudades y comunidades sostenibles.

Meta 11.1. Asegurar el acceso a viviendas y servicios adecuados; mejorar los barrios marginales.

Meta 11.5. Reducir el número de muertes y de personas afectadas por los desastres, así como las pérdidas económicas vinculadas.

- Objetivo 13. Acción por el clima. 
Meta 13.1. Resiliencia frente a los riesgos relacionados con el clima y los desastres naturales.

- $\quad$ Objetivo 16. Paz, justicia e instituciones sólidas.

Meta 16.1. Reducir la violencia y las correspondientes tasas de mortalidad.

Meta 16.2. Poner fin al maltrato, la explotación, la trata, la violencia y la tortura contra los niños.

- Objetivo 17. Alianzas para lograr los objetivos.

Meta 17.16. Fortalecer alianzas mundiales entre múltiples interesados para promover el logro de los Objetivos de Desarrollo Sostenible en todos los países.

Meta 17.18. Asistir a los países en desarrollo para aumentar la disponibilidad de datos de gran calidad, desglosados por situación migratoria (OIM, 2018).

En particular, la Meta 10.7, que aborda la migración en el contexto más amplio de la reducción de las desigualdades de toda índole [ODS 10], refleja como imperativo para todos los Estados el mejoramiento de la gestión y la planificación de sus políticas migratorias. Ello supone la supervisión y la medición de los progresos hacia la consecución de los Objetivos de Desarrollo Sostenible (ODS) a escala nacional, tomando en cuenta sus múltiples dimensiones (tanto cualitativas como cuantitativas), en el entendido de que los marcos de gobernanza migratoria deben evaluarse bajo tres vertientes: su inversión en las personas; su eficacia para la creación de capacidad institucional en los Estados (coherencia política y cobertura de las políticas); y su éxito en lo que respecta a abrir más vías para la migración legal. Por ende, corresponde asegurar la existencia de un marco normativo que promueva la migración segura, ordenada y regular; verificar que las medidas y las metodologías aplicadas sean adecuadas para los fines previstos; definir los indicadores que se van a medir y los términos en que se describen las metas y los objetivos a alcanzar; y generar nuevas fuentes de datos desagregados en función del estatus migratorio, el género, la edad y otros factores pertinentes (OIM, 2017, pp. 13-14).

En este sentido, la Organización Internacional para las Migraciones (OIM), ateniéndose a la condición de organismo intergubernamental por ser una agencia conexa de la Organización de Naciones Unidas (ONU), apoya a sus asociados de la comunidad internacional para encarar los retos planteados por la gestión de la migración. De ahí el monitoreo que viene haciendo de las metas específicas que enmarcan a ésta en los Objetivos de Desarrollo Sostenible (ODS), amén de acompañar las negociaciones intergubernamentales con miras al «Pacto Mundial para una Migración Segura, Ordenada y Regular», que se adoptará en 2018.

En este proceso, cobra notabilidad el «Marco de la Gobernanza de la Migración» (MIGOF) aprobado por el Consejo de Países Miembros de la Organización Internacional para las Migraciones (OIM) mediante Resolución $\mathrm{N}^{\circ} 1310$ del 24 de noviembre del 2015, el cual encarna un valioso instrumento de cara a la administración de evaluaciones de país con base en un conjunto de tres principios y tres objetivos coherentes, abarcadores y equilibrados, en concreto: 
- Principios:

1. Adhesión a las normas internacionales y respeto de los derechos de los migrantes.

2. Formulación de políticas contrastadas y aplicación de enfoques de gobierno integrados.

3. Colaboración con sus asociados para hacer frente a la migración y a las cuestiones conexas.

- Objetivos:

1. Fomentar el bienestar socioeconómico de los migrantes y de la sociedad.

2. Abordar eficazmente los aspectos relativos a la movilidad en situaciones de crisis.

3. Velar porque la migración se efectúe de manera segura, ordenada y digna (OIM, 2017b).

Otro tanto entraña el denominado «Índice de la Gobernanza Migratoria» (MGI), desarrollado por la Organización Internacional para las Migraciones (OIM) en asociación con The Economist Intelligence Unit, con el propósito de ayudar a los gobiernos a medir el performance de sus estructuras de gobernanza de las migraciones, atendiendo a sus prioridades, progresos y deficiencias en cinco diferentes esferas normativas:

1. Capacidad institucional.

2. Derechos de los migrantes.

3. Migración laboral.

4. Gestión de una migración segura y ordenada.

5. Cooperación regional e internacional y otras alianzas (OIM, 2017, p. 23).

Aparte de estas herramientas, la Organización Internacional para las Migraciones (OIM) despliega su acción a través del «Diálogo Internacional sobre la Migración», instancia fundada en 2001 a petición de los Estados miembros del organismo, en la vía de emplazar un foro que auspicie una mayor comprensión de los problemas de la migración internacional; el establecimiento de redes de expertos de distintas esferas y regiones, junto a responsables de la formulación de políticas públicas, para intercambiar puntos de vista normativos y prácticas eficaces en el futuro; y consolidar la cooperación entre los gobiernos con otros interlocutores tratándose de esta materia. No en balde, en 2016 convocó dos talleres centrados en el examen de la Agenda 2030 para el Desarrollo Sostenible y, en particular, sobre las metas relativas a la migración en el contexto de los 17 Objetivos de Desarrollo Sostenible (ODS). El primer taller tuvo lugar en la sede de la Organización de Naciones Unidas (ONU), en Nueva York, del 29 de febrero al 1 de marzo, mientras que el segundo se llevó a cabo en Ginebra, los días 11 y 12 de octubre (OIM, 2017, p. 3).

Con todo, cada una de las instancias antes mencionadas ha labrado el camino al Pacto Mundial sobre Migración, en el cual se espera sean fijados los principios y los enfoques para gestionar de forma innovadora los flujos migratorios a nivel internacio- 
nal durante las siguientes décadas, y fomentar una mayor coherencia entre las políticas migratorias de cada uno de los Estados.

\section{Principales tendencias migratorias en Iberoamérica}

Según la Organización Internacional para las Migraciones -OIM- (2017), la mayoría de los migrantes internacionales permanecen en su región de origen, perfilándose la migración Sur-Sur como el componente geográfico de crecimiento más rápido de la movilidad humana, a pesar de que, como tendencia, las corrientes siempre han estado definidas por el tránsito desde los países en desarrollo hacia los países desarrollados de Europa, América del Norte y Australasia (PNUD, 2009) En este enmarcado, la preocupación por las migraciones y el refugio se ha disparado de manera exponencial en Iberoamérica, atisbándose la diferencia del desarrollo entre los países de origen, tránsito y de recepción como la causa de los llamados «puentes o corredores migratorios» (Fernández et al., 2017).

No por casualidad, la sucesión de Cumbres Iberoamericanas celebradas desde 1991, entendidas como mecanismo regional para el encuentro y el diálogo, ha creado un espacio para la discusión de las principales preocupaciones y desafíos en materia migratoria tratándose del ámbito latinoamericano. En este sentido, especialmente significativa puede considerarse la cita realizada en Montevideo (Uruguay) en noviembre de 2006, la cual derivó en el "Compromiso de Montevideo sobre Migraciones y Desarrollo», a la postre un valioso instrumento político orientador de la acción toda vez que señala la relevancia de las migraciones para la comprensión de la historia de esta región del mundo, valorando positivamente sus aportes sociales e interculturales, sin menoscabo de las contribuciones a las economías de los países de acogida (Universidad Pontificia de Comillas y OBIMID, 2017).

A raíz del «Compromiso de Montevideo» surgieron las primeras dos ediciones del Foro Iberoamericano sobre Migración y Desarrollo (FIBEMYD), llevadas a cabo en Cuenca (Ecuador, 2008) y San Salvador (El Salvador, 2010). Ambas iniciativas serían refrendadas por la Conferencia Sudamericana de Migraciones (CSM) efectuada en 2010, sirviendo de telón de fondo para la aprobación del «Plan Sudamericano de Desarrollo Humano de las Migraciones, contexto y perspectivas», el cual anida en una concepción de la gobernabilidad migratoria centrada en los Derechos Humanos de los migrantes, sea cual sea su origen nacional, religioso o étnico; la no criminalización en función de su situación administrativa migratoria, y la no utilización como variable de ajuste de las economías o mercados de trabajo.

Paralela a estos esfuerzos, la creciente inmigración en los países de América Latina y el Caribe ha respondido a un carácter intrarregional en razón de los acuerdos regionales de integración, pero también por las crisis económicas y políticas que enfrentan los países en distintos momentos de su historia (OIM y CEPAL, 2018). En esta tónica, según el informe de la Organización de Estados Americanos (OEA) de 2015, hasta ese año la zona había albergado 9,2 millones de migrantes, de donde la mitad migra hacia Estados Unidos y Canadá, una cuarta parte se traslada hacia otras regiones, y la otra cuarta parte se mueve entre otros países de América. 
Mesoamérica y el Caribe se consideran subregiones de origen y tránsito, aun cuando los flujos migratorios irregulares provenientes de Cuba y Haití, así como de los países asiáticos y africanos han aumentado allí significativamente. Los países que más recientemente han atraído flujos intrarregionales, como México, Belice, El Salvador, Panamá y Trinidad y Tobago, se han consolidado como países de destino (OIM, 2017b).

Por lo que atañe a Suramérica, desde la implementación del Acuerdo de Residencia del Mercosur (2009) se han otorgado más de dos millones de residencias temporales y permanentes a nacionales de la región, posicionándose Argentina como el principal país receptor de los flujos de inmigrantes de la región, secundada por Chile y Brasil. Los trabajadores representan la mayor parte de la migración en sectores con altos niveles de empleo poco cualificados, sometidos a condiciones irregulares y precarias (agricultura, construcción, trabajo doméstico y por cuenta propia). Recientemente, los países de América del Sur han recibido un número creciente de migrantes extra-regionales provenientes de África, Asia, Europa, Estados Unidos y el Caribe. En cuanto a la emigración extra-regional, Estados Unidos, España y otros países europeos y, en menor medida, Japón, son los principales países de destino (OIM, 2017b).

Actualmente, en la subregión destaca la situación por la que atraviesa la República Bolivariana de Venezuela, ateniéndose a las alteraciones verificadas en su tradición migratoria. En esta línea, si bien sigue siendo el segundo país receptor neto de población, al mismo tiempo registra un importante crecimiento de su emigración, la cual prácticamente se duplicó durante el período intercensal 2000-2010 hacia distintos países, encabezando la lista Estados Unidos y España como los principales destinos (185 000 y 155000 personas, respectivamente), sin descartar a Colombia, Ecuador, Panamá, México, Costa Rica, Argentina y Chile (OIM y CEPAL, 2018). Desde 2012, el clima de conflictividad política y social se ha intensificado, con el agravante de que el país ha entrado nuevamente en una fase de contracción económica, con altos niveles de endeudamiento, escasez de productos básicos e inflación que han incidido fuertemente sobre la capacidad adquisitiva de la población (Universidad Pontificia de Comillas y OBIMID, 2017).

Curiosamente, el gobierno de Venezuela aprobó una Ley de Extranjería y Migración en 2004, enunciando el compromiso de cumplir con los instrumentos suscritos y ratificados en los diversos escenarios internacionales y procesos de integración de los cuales el país forma parte; empero, el documento no contempla ningún título (o capítulo) referido al registro de los venezolanos residentes en el exterior y a la protección de sus derechos, lo que deviene en la carencia de un plan de actuación frente a la diáspora (Fernández, et. al., 2017). Desde este punto de vista, las esferas gubernamentales en Venezuela no han otorgado reconocimiento a la creciente comunidad transfronteras, ora porque ello entrañaría admitir que el modelo político y económico genera emigrantes, ora porque la organización política de venezolanos en el extranjero genera mala imagen para el régimen -por ejemplo, las protestas encabezadas por grupos en distintas ciudades del mundo, denunciando la falta de libertades civiles y políticas: los famosos «escraches» (BBC Mundo, 2017)-. Este hecho ha permitido a las instancias oficiales politizar el tema y ubicarlo en un discurso nacionalista que identifica al país y su soberanía con el proyecto socialista, visualizándose la migración, en un contexto simbólico, como una «válvula de escape» frente a presiones internas -exportar la opo- 
sición-, de donde se sigue la legitimación de su exclusión y la justificación moral de su represión -traidores a la patria/aliados de un enemigo extranjero- (Bobes, 2012).

No en vano, desde 2004 el Alto Comisionado de las Naciones Unidas para los Refugiados (ACNUR) ha venido registrando el incremento de la población venezolana solicitante de protección en otros países, a la que se le ha otorgado el status de refugiado o se encuentra en una situación similar a refugiado. En este contexto, el ACNUR insta a los Estados receptores y/o a los que ya acogen a los venezolanos para que adopten mecanismos orientados a brindar protección internacional en tanto acto humanitario consistente con el espíritu de solidaridad subyacente en la Convención sobre el Estatuto de los Refugiados de 1951 y la Declaración de Cartagena. Reconociendo este desafío, los estándares mínimos han de garantizarse bajo todas las circunstancias, específicamente legalidad, accesibilidad, derechos básicos (atención médica, educación, unidad familiar, libertad de circulación, acceso a albergue y derecho al trabajo), y garantías de no retorno (ACNUR, 2018).

En últimas, la radicalización ideológica escenificada durante los últimos años en Venezuela, junto a la polarización política, ha devenido en un desdén por los emigrados y la aplicación de una "política de la no política» como saldo por haber abandonado el país y el proyecto político liderado por Hugo Chávez, continuado desde 2013 por Nicolás Maduro (Muñoz, 2016). Para el Secretario General de Amnistía Internacional (AI), Salil Shetty, la «crisis humanitaria y de Derechos Humanos» que se vive en Venezuela «acecha» a Suramérica, lo que llama a una solución «política» y «regional».

\section{A modo de cierre}

Gestionar la migración de un modo eficaz implica asumir un enfoque holístico que favorezca el tratamiento humano de los migrantes -independientemente de su estatus migratorio-, poniendo en primer plano la totalidad de sus dimensiones y aspectos esenciales como personas.

En la actualidad, no son pocos los llamados para crear un sistema mundial de gobernanza de la migración que permita a los gobiernos enfrentar desafíos de manera compartida y generar respuestas en conjunto, instalando el desarrollo humano en la agenda de las políticas públicas para incrementar los beneficios a partir de los patrones cada vez más complejos del movimiento humano en el mundo. En este orden de ideas, no resulta desatinado aseverar que, en adelante, deberán considerar la integración de la migración en sus respectivos procesos e instrumentos de planificación, como los planes locales, nacionales y regionales de desarrollo.

$\mathrm{El}$ «cómo» y el «cuándo» de las reformas dependerá de un examen realista de las condiciones económicas y sociales de cada país, en el entendido de que la migración constituye un fenómeno multidimensional y transnacional en el que todos, sin distingo, son emisores, receptores e, incluso, lugares de tránsito para las personas. De allí que la cooperación internacional, por medio de acuerdos bilaterales o regionales, puede redundar no sólo en una protección más eficiente de los derechos de los migrantes, sino también en el aprovechamiento de su potencial y, con ello, el aumento de sus contribuciones tanto al desarrollo de los países y las comunidades de origen como al 
desarrollo de los países y las comunidades de acogida, en el marco de un proceso de globalización inclusivo.

Sin embargo, no incumbe únicamente a los gobiernos la responsabilidad por la consecución de estos objetivos; antes bien, los logros estarán sujetos, en gran medida, a su capacidad para incorporar a otros interlocutores en el proceso, como el sector empresarial, la sociedad civil y las comunidades de la diáspora. En el caso del primero, su aporte es evidente por tratarse de uno de los principales motores de creación de valor a largo plazo en términos económicos, siendo las compañías privadas las principales empleadoras para la mayoría de los migrantes que se desplazan en busca de puestos de trabajo, amén de prestar servicios de contratación, viajes y transferencia de fondos. Desde esta óptica, las empresas no habrán de tolerar la discriminación por razones de raza, origen étnico o religión, ni en los recintos laborales ni en la cadena de suministro, evitando además las prácticas de explotación. Asimismo, deberán respetar los derechos de los trabajadores migrantes a la libertad de asociación y a entablar negociaciones colectivas, concienciando las ventajas que supone la incidencia de la diversidad sobre aspectos como la innovación, los nuevos mercados y la productividad (MF Saltaji, 2017; OIM, 2017).

Por lo que concierne a la sociedad civil, la aceptación de los migrantes representa una oportunidad para nuevas interacciones en función de un enriquecimiento mutuo, identificando y superando, merced campañas de sensibilización, las fuentes de hostilidad y de tensiones sociales. No en vano, la otrora «invisibilidad» de los migrantes, que conllevaba dejar de lado la consideración de sus derechos, su dignidad y sus necesidades, ha dado paso en la actualidad a una atención sostenida fundada en estereotipos, estigmas y prejuicios generalizados.

Finalmente, pero no por eso menos relevante, destaca el rol desempeñado por las diásporas a la luz de su potencial como «puentes» entre sociedades y agentes del desarrollo, toda vez que facilitan el acceso a ideas, conocimientos y recursos que sirven de complemento y, en algunas ocasiones, logran intensificar el progreso los países de origen (PNUD, 2009). Por consiguiente, se sigue un estudio más sistemático del perfil de la migración y de sus beneficios, costos y riesgos por parte de los Estados con salidas significativas de población hacia el exterior, aunado a la habilitación de un departamento o ministerio dedicado a asuntos de la diáspora, sin descontar la asunción de políticas nacionales que ofrezcan apoyo y defiendan los intereses de las comunidades migrantes apelando a la protección consular y los servicios jurídicos (El Vaticano, 2017).

No obstante, el conocimiento de las diásporas no basta para promover la colaboración; antes bien, las estrategias de participación eficaces demandan la creación de confianza (OIM, 2017). Se requiere la adopción de diferentes mecanismos para conocer cuánta población se ha establecido fuera del territorio nacional y en qué circunstancias se encuentra; vincularse con las comunidades en el exterior y promover actividades que contribuyan a la preservación de sus nexos con el país.

Estas consideraciones coinciden y están en sintonía con los planteamientos de organismos internacionales en aras de fomentar la comprensión en torno a la dinámica de la migración, las condiciones en las que se produce, junto a las oportunidades y las exigencias que trae consigo. De por sí, el reconocimiento oficial del aporte de la migración a los Objetivos de Desarrollo Sostenible (ODS) encarna un gran avance, 
impulsando el diseño de estrategias de integración con impacto real sobre la vida de los 244 millones de migrantes internacionales que hay en el mundo, a la par que promueve los debates regionales y mundiales en pro de políticas migratorias cada vez más planificadas y mejor gestionadas.

\section{Referencias}

ACNUR (2018). “Nota de orientación sobre el flujo de venezolanos”. (https://goo.gl/Rbmk1d) (2106-2018).

BBC Mundo (2017, 3 de mayo). Los "escraches" contra figuras asociadas al gobierno de Venezuela. El Nacional. (https://goo.gl/7mpWuw) (14-07- 2017).

Bobes, V. (2012). Diáspora, ciudadanía y contactos transnacionales. Nueva Sociedad, (242), 106122.

Castillo, C. (2015). El rostro humano de la migración. Diálogo Político, 32(2), 96-103.

El Vaticano (2017). Responder a los desafíos de los refugiados y migrantes. Veinte puntos de acción. Migración y Desarrollo, 15(29), 123-135.

Fernández, M., et al. (2017). Estudio: migración y desarrollo en Iberoamérica. Informe Ejecutivo. España: OIM-SEGIB.

Global Forum on Migration and Development GFMD Turkey 2014-2015. Mesa redonda 2: La migración como factor de desarrollo. Mesa redonda 2.2 La migración en el marco de la Agenda 2030 para el Desarrollo: seguimiento y examen de una cuestión polifacética y transversal en pos de su incorporación. (https://goo.gl/EaSdVE) (21-06-2018).

MF Saltaji, I. (2017). Corporate Social Responsability and migration: integration governance. Internal Auditing $\mathcal{E}$ Risk Management, 12(48), 58-69.

Muñoz, T. (2016). Políticas migratorias en México y Venezuela: análisis de respuestas gubernamentales disímiles ante procesos de inmigración y emigración internacionales. Desafíos, 28(2), 333-366. DOI: http://dx.doi.org/10.12804/desafios28.2.2016.09

OIM (2017a). Seguimiento y evaluación de los aspectos referentes a la migración en los Objetivos de Desarrollo Sostenible. Diálogo Internacional sobre Migración N²6. Ginebra: OIM.

OIM (2017b). "La OIM y la Agenda 2030 para el desarrollo sostenible en América Latina y el Caribe". (https://goo.gl/YdrrC1) (15-05-2018).

OIM (2018). "La migración y los objetivos de desarrollo sostenible. Reunión técnica de socios de la iniciativa conjunta de salud para las personas migrantes". (https://goo.gl/tvTpjK) (15-05-2018).

OIM y CEPAL (2018). Informe final de la reunión regional latinoamericana y caribeña de expertos y expertas en migración internacional preparatoria del Pacto Mundial para una migración segura, ordenada y regular. Santiago de Chile: OIM y CEPAL.

Pinazo, J. (2017). Inmigración en el estado postwestfaliano: un enfoque de políticas públicas. BARATARIA Revista Castellano-Manchega de Ciencias Sociales, (23), 129-143. DOI: http://dx.doi. org/10.20932/barataria.v0i23.345

PNUD (2009). Informe sobre Desarrollo Humano 2009. Nueva York: PNUD.

Universidad Pontificia de Comillas y OBIMID (2017). Estudio: migración y desarrollo en Iberoamérica. España: Universidad Pontificia de Comillas y OBIMID.

Foro Económico Mundial (2018). Informe de riesgos mundiales 2018. Ginebra: Foro Económico Mundial (13. ${ }^{\mathrm{a}}$ edición). 